\title{
Management of failed spinal anaesthesia for caesarean section
}

\author{
G W Jones, ${ }^{1}$ MB BCh, DA (UK), FRCA (UK), MBA, FCA (SA); R A Samuel, ${ }^{2}$ MB ChB, DA (SA), FCA (SA), MMed; \\ B M Biccard, ${ }^{3} \mathrm{MB}$ ChB, FCA (SA), FFARCSI, MMedSci, PhD \\ ${ }^{1}$ Department of Anaesthesia, Faculty of Health Sciences, University of KwaZulu-Natal, Durban, South Africa \\ ${ }^{2}$ Private Practice, Durban, South Africa \\ ${ }^{3}$ Department of Anaesthesia and Perioperative Medicine, Faculty of Health Sciences, Groote Schuur Hospital and University of Cape Town, South Africa
}

Corresponding author: GW Jones (gavin@jonesmail.co.za)

Background. Failed spinal anaesthesia for caesarean section (CS) may be partial or complete and the subsequent discomfort is the most commonly cited cause of litigation in obstetric anaesthesia.

Objectives. To determine if there is a standardised approach to: (i) testing the level of block of spinal anaesthesia; and (ii) the management of failed spinal anaesthesia for CS.

Methods. A structured questionnaire to ascertain the current practice of testing the level of block and management of three different scenarios of failed spinal anaesthesia was distributed to 51 government hospitals in KwaZulu-Natal, South Africa (SA). All obstetric anaesthetic service providers, ranging from interns to specialist anaesthetists, were invited to complete the questionnaire.

Results. A total of 375 responses were received from 42 of the 51 hospitals surveyed. Specialist anaesthetists managed failure of spinal anaesthesia significantly differently than other anaesthetic service providers. Specialists were more likely to convert to a general anaesthetic (GA), while others were more likely to repeat spinal anaesthesia or administer intravenous ketamine, midazolam and opioids. Only 212 respondents (56\%) tested the level of block and there was no difference between the groups with regard to the method of assessment of height ( $p=0.15)$. Nonspecialists, however, accepted a significantly lower level of block, using pinprick, than specialists $(p=0.027)$, which could lead to a higher failure rate. More than one-third of non-specialists did not consider themselves competent to perform a GA and $>90 \%$ of respondents agreed that a 'failed' spinal algorithm would be useful.

Conclusion. There is a need for standardised assessment of the adequacy of spinal anaesthesia for CS in SA, as well as a failed spinal algorithm.

S Afr Med J 2017;107(7):611-614. DOI:10.7196/SAMJ.2017.v107i7.12056

Spinal anaesthesia has become the preferred choice of anaesthetic for caesarean section (CS) owing to rapid onset, predictable and reliable block and excellent postoperative analgesia, as well as the avoidance of the risks of a general anaesthetic (GA), such as airway difficulties and neonatal drug toxicity. ${ }^{[1,2]}$

Managing spinal anaesthesia that is inadequate for surgery can be extremely challenging; doctors need to be aware of all the possible reasons for failure. The anaesthetic technique must maximise the chance of success.

Failure of regional anaesthesia is defined in a number of ways: ${ }^{[3]}$

- pain during surgery

- conversion to a GA

- conversion to any other form of anaesthesia

- inability to achieve a defined degree of nerve block adequate for CS (epidural top-up).

Failed spinal anaesthesia may be partial or complete and may require administration of various adjuvants or conversion to a GA, which may have medicolegal implications. The most commonly cited cause of litigation in obstetric anaesthesia is discomfort during spinal anaesthesia for CS. ${ }^{[1]}$

In the Saving Mothers Report, ${ }^{[4]} 73$ (79\%) of the 92 assessed deaths relating to anaesthetic complications were due to spinal anaesthesia, based on an intent-to-treat analysis. Of these, $10(14 \%)$ were patients who died of complications of a subsequent GA, which was administered as the spinal anaesthesia proved inadequate for surgery.
It is possible that a systematic, algorithmic approach to the management of inadequate spinal anaesthesia would have reduced the need to convert to a GA and, if the conversion had been necessary, it would have proved less hazardous.

In South Africa (SA) there is currently no standard approach to testing the level of a spinal block, nor is there an algorithm on how to manage a spinal anaesthetic block that is inadequate for surgery. A clearer picture of how different grades of clinicians currently manage failures may assist the development of a practical algorithm applicable to the SA context, which may lead to improved patient management and reduced perinatal and maternal morbidity and mortality.

The objectives of this study were to: (i) determine if there is a standardised approach to the management of a failed spinal anaesthetic for CS; (ii) determine the modalities used for testing the level of block and what level of block was deemed adequate; (iii) assess how many anaesthetic service providers follow the UK standard of care; and (iv) determine whether the UK's 'inadequate regional anaesthesia' guidelines ${ }^{[5]}$ are appropriate for SA.

\section{Methods}

This was a descriptive, observational cross-sectional study. All obstetric anaesthetic service providers, including specialist anaesthetists, medical officers, registrars, community-service doctors and interns working in 51 government hospitals in KwaZulu-Natal (KZN), SA, were invited to complete a simple questionnaire, ${ }^{\star}$ which was structured to 
ascertain current practice of testing the level of block and the management of three different scenarios of failure of spinal anaesthesia. There were no exclusions. For each scenario, the respondents were asked to rank their top three management choices, where each subsequent intervention would be chosen if the prior intervention was inadequate. The respondents could choose between 12 management options (Table 1).

Scenario 1 consisted of the management of an elective CS with loss of cold sensation to a level of T6 after 30 minutes, but lifting one leg, prior to skin incision. Scenario 2 comprised an elective CS with significant pain immediately on skin incision. Scenario 3 was an emergency CS with pain on incision of the uterus.

Questionnaires were hand delivered or emailed and responses collected over a period of 3 months, from May to July 2014.

All categorical data were analysed using descriptive statistics and presented as percentages and 95\% confidence intervals, where appropriate. Categorical data were analysed using Fisher's exact test or Pearson's $\chi^{2}$ test, where appropriate. SPSS version 23.0 (IBM Corp., USA) was used for data analysis.

Approval was obtained from the Biomedical Research Ethics Committee of the University of KZN (ref. no. BE 370/13) and the Health Research and Knowledge Management sub-component of the KZN Department of Health (ref. no. HRKM 32/14).

\section{Results}

Of the 51 hospitals invited to participate, 42 accepted. These hospitals included tertiary, regional and district hospitals; 375 anaesthetic service providers participated in the study. The grades of the anaesthetic service providers are shown in Table 2.

The results of the first objective to determine if there is a standardised approach to the management of failed spinal anaesthesia for CS are given below.

\section{Scenario 1: Management of an elective CS with loss of cold sensation to $\mathrm{T} 6$ after 30 minutes, but lifting one leg, before skin incision}

The top three management choices as the first intervention to manage this scenario were to change position (32.8\%), repeat spinal

\begin{tabular}{ll}
$\begin{array}{l}\text { Table 1. Management options for the treatment of } \\
\text { inadequate spinal anaesthesia }\end{array}$ \\
\hline Option number & Management options \\
\hline 1 & Ketamine IV increments \\
2 & Ketamine/midazolam mix IV increments \\
3 & Short-acting opioids (e.g. alfentanyl) \\
& IV increments \\
4 & Long-acting opioids (e.g. morphine) \\
5 & IV increments \\
6 & $\mathrm{~N}_{2} \mathrm{O} / \mathrm{O}_{2}$ mix \\
7 & Changing position \\
8 & Repeat spinal - full dose \\
9 & Repeat spinal - partial dose \\
10 & Epidural \\
11 & Convert to a GA \\
12 & Postpone CS for 24 hours \\
IV = intravenous; GA = general anaesthetic; $\mathrm{N}_{2} \mathrm{O}=$ nitrous oxide; CS = caesarean section.
\end{tabular}

anaesthesia with a partial dose (14.4\%), and convert to a GA $(9.1 \%)$. The overall ranking of management choices that would be considered as one of the first three interventions was to convert to a GA (16.7\%), change position $(12.9 \%)$, and repeat spinal anaesthesia with a partial dose $(9.2 \%)$. Sixteen respondents $(4.3 \%)$ would have continued with the CS without any further action.

The management by specialist anaesthetists compared with other anaesthetic service providers was significantly different $(p<0.001)$; they were more likely to convert to a GA, while non-specialists were more likely to repeat the spinal anaesthesia.

\section{Scenario 2: Management of an elective CS with significant pain immediately on skin incision}

The top three management choices as the first intervention to manage this scenario were to convert to a GA (31.7\%), repeat spinal anaesthesia with a full dose (16.3\%), and administer ketamine intravenously (IV) in increments $(11.5 \%)$. The overall ranking of management choices that would be considered as one of the first three interventions was to convert to a GA (24.2\%), administer ketamine IV in increments (7.5\%), and repeat spinal anaesthesia with a full dose (7.4\%).

The management by specialist anaesthetists compared with other anaesthetic service providers was significantly different $(p<0.001)$; they were more likely to convert to a GA, while non-specialists were more likely to repeat the spinal anaesthesia at a full dose.

\section{Scenario 3: Management of an emergency CS with pain on incision of the uterus}

The top three management choices as the first intervention to manage this scenario were to administer ketamine IV in increments

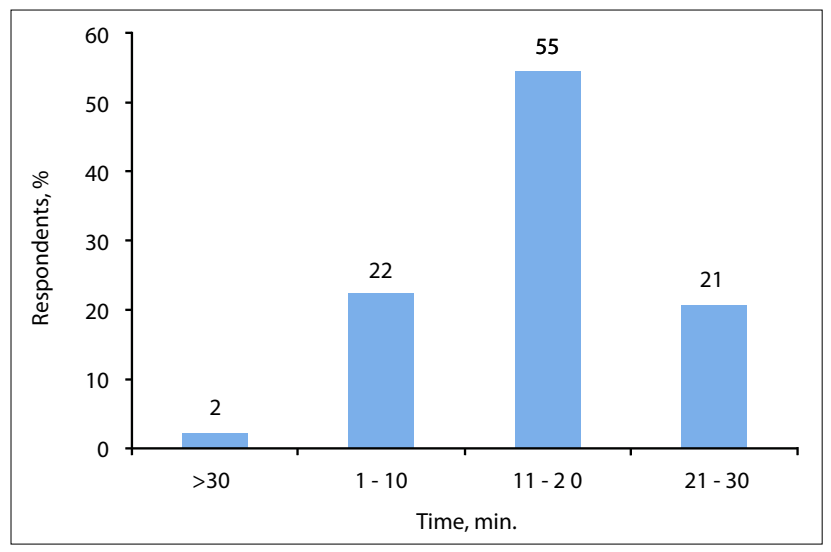

Fig. 1. Time respondents waited before declaring the block inadequate for surgery.

Table 2. Grade of participating anaesthetic service providers

\begin{tabular}{ll}
\hline Grade & $\boldsymbol{n}(\%)$ \\
\hline Intern & $17(4.5)$ \\
Community service & $43(11.5)$ \\
Medical officer <2 years & $64(17.1)$ \\
Medical officer $>2$ years & $90(24.0)$ \\
Medical officer and DA & $47(12.5)$ \\
Anaesthetic registrar & $54(14.4)$ \\
Specialist anaesthetist & $56(14.9)$ \\
Unknown & $4(1.1)$ \\
Total & $375(100)$ \\
DA = Diploma in Anaesthetics. &
\end{tabular}


(22.7\%), convert to a GA (20.8\%), and administer an $\mathrm{N}_{2} \mathrm{O} / \mathrm{O}_{2}$ mix (16.5\%). The overall ranking of management choices that would be considered as one of the first three interventions was to convert to a GA (21.3\%), administer ketamine IV incrementally (13.2\%), and administer an $\mathrm{N}_{2} \mathrm{O} / \mathrm{O}_{2} \operatorname{mix}(10.3 \%)$.

The management by specialist anaesthetists compared with other anaesthetic service providers was again significantly different $(p=0.03)$; they were more likely to convert to a GA, while nonspecialists were more likely to administer ketamine, midazolam and long-acting opioids.

The results of the second objective to determine the modalities used for testing the level of block and what level of block was deemed adequate for CS are as follows: The respondents did not routinely test the level of a spinal anaesthesia block, with $212(56 \%)$ respondents testing the level of block and 33 of the $56(59 \%)$ specialists testing spinal anaesthesia block levels. The method of assessment of block height varied among respondents. Of those who used: ( $i$ ) light touch, 55\% accepted a block level of T6-8; (ii) temperature, $41.7 \%$ accepted a level of T4-6; (iii) pinprick, $42.9 \%$ accepted a level of T8 - 10; and (iv) a motor block alone to determine the adequacy of spinal anaesthesia, $10.8 \%$. The majority of the respondents waited 11 - 20 minutes before declaring the block inadequate for surgery (Fig. 1). There was no difference between specialist anaesthetists and other anaesthetic service providers regarding the method of assessment of block height $(p=0.15)$.

The spinal anaesthesia block levels considered acceptable for surgery are shown in Table 3. There was a significant difference between anaesthetic specialists and other anaesthetic service providers with regard to the height of block considered acceptable $(p<0.001)$. Anaesthetic specialists considered higher blocks adequate (T4 - 6, 48.2\%; T6 - 8, 41.1\%) compared with non-specialist anaesthetic providers (T4-6, 25.1\%; T6 - 8, 35.7\%; T8 - 10, 27.0\%).

While there was no significant difference in the level of block for temperature and light touch between specialists and non-specialists for the assessment of block height using pinprick, the non-specialists accepted a significantly lower level of block than the specialists $(p=0.027)$ (Table 4).

More than a third of non-specialist anaesthetic providers $(36.7 \%)$ did not believe that they were competent to provide a GA for CS

Table 3. Reported acceptable spinal anaesthesia block levels for caesarean section

\begin{tabular}{ll}
\hline Level & $\boldsymbol{n}(\%)$ \\
\hline Not reported & $4(1.9)$ \\
T2 - 4 & $4(1.9)$ \\
T4 - 6 & $66(31.1)$ \\
T6 - 8 & $87(41.0)$ \\
T8 - 10 & $51(24.1)$ \\
Total & $212(100)$
\end{tabular}

Table 4. Reported acceptable spinal anaesthesia block levels when using pinprick for caesarean section

\begin{tabular}{lll}
\hline Level & Non-specialists, \% & Specialists, \% \\
\hline T4 - 6 & 18.2 & 66.7 \\
T6 - 8 & 27.3 & 33.3 \\
T8 - 10 & 54.5 & 0
\end{tabular}

compared with $100 \%$ of specialists, who considered themselves competent to provide a GA $(p<0.001)$, and $92.5 \%$ of respondents thought a 'failed' spinal algorithm would be useful, with no difference between specialist and non-specialist providers $(p=0.24)$.

\section{Discussion}

The main findings of this study are that the interventions for managing inadequate spinal anaesthesia for CS differ between anaesthesia service providers in SA, that there is no universal agreement on the modality of testing block height and the acceptable block height for anaesthesia, and that while specialists are more likely to convert to a GA and feel competent in this task, non-specialists are less likely to convert to a GA and more than one-third of them do not consider themselves competent to perform a GA for CS. More than $90 \%$ of respondents agree that a failed spinal algorithm would be useful.

This study raises a number of concerns regarding CS anaesthesia in SA.

There is a lack of uniformity in the assessment of the adequacy of spinal anaesthesia for CS, both in modality used and the height considered acceptable. Only 56\% tested the adequacy of spinal anaesthesia. The four modalities used to test were touch, sharp pinprick, cold and motor. Generally, loss of sensation to touch is several dermatomes lower than pinprick, which in turn is several dermatomes lower than cold. This is important in clinical practice, as the anaesthetist needs to be aware of the implications of an assumed adequate level of block, with subsequent discomfort or pain for the patient. There is also great variability between and in patients with regard to the three different modalities. ${ }^{[6]}$

The level required for skin incision for CS is the T10 dermatome. However, a number of visceral organs send sympathetic afferent impulses to the thoracic spinal cord and require a block level to T4. If this is not achieved, a dull, cramping visceral-type pain is often felt, leading to a 'failed' spinal. Russell ${ }^{[7]}$ has shown that when the block to touch is lower than T5, patients often feel pain. Surprisingly, in these patients the corresponding block to cold or pinprick was much higher than T4 and often at the cervical level. Accordingly, Russell ${ }^{[7]}$ states that if T5/6 is the required block level to touch, then a block level to pinprick and cold of two and four dermatomes higher, respectively, should be obtained.

Our study indicates that the majority of respondents wait an acceptable time to declare inadequacy of the spinal anaesthesia block. Fettes et al. ${ }^{[8]}$ suggest that one should allow 15 - 20 minutes after insertion before determining that the spinal anaesthetic has failed.

A major concern is that more than one-third of the non-specialist anaesthetic providers stated that they did not feel competent to provide a GA for CS. This may, therefore, explain why this group, when compared with specialist anaesthetists, provided significantly more 'non-GA' interventions for inadequate spinal anaesthesia. It is likely that these anaesthesia providers may find themselves in an unenviable position of reluctantly administering a GA, once they have exhausted all non-GA alternatives. This may lead to an even more hazardous subsequent GA. There was an almost universal appeal for a failed spinal algorithm in this study.

A search of the literature revealed very few failed spinal algorithms, and the study has highlighted that in KZN there is no standard approach to managing a spinal anaesthesia that has failed.

The Royal College of Anaesthetists suggests that, in keeping with best practice, the conversion rate from spinal anaesthesia to a GA should be $<1 \%$ for elective CS and $<3 \%$ for non-elective CS. ${ }^{[9]}$ Some of the common reasons stated for failure to meet these standards include: ${ }^{[9]}$ - lack of a dedicated obstetric anaesthetist and inexperienced staff 
- misunderstanding/misclassifying urgency and poor selection of regional anaesthesia type in complex cases

- inappropriate assessment/recording of the block.

This study suggests that staff inexperience and inappropriate assessment of the spinal anaesthesia block may be a major factor in SA. We did not assess an understanding of urgency or the appropriateness of the selection of regional anaesthesia. It is likely, however, that based on the reported lack of competence in administering a GA for CS, it is possible that inappropriate selection of spinal anaesthesia for CS is also a concern in SA.

Spinal anaesthesia in inexperienced hands is associated with significant maternal mortality. ${ }^{[4]}$ The first part of the title of a recent article by Farina and Rout, ${ }^{[10]}$ 'But it's just a spinal: ..., aptly summarises the attitude of many doctors. There is a misconception that spinal anaesthesia can be performed safely by a doctor lacking GA skills in advertisements for medical officers in SA. One district hospital, for example, listed 'perform spinal anaesthesia' as a key competency. A more appropriate competency would be 'capable of providing anaesthesia' and include all forms of anaesthesia relevant to the procedures required to be performed at a district hospital. ${ }^{[10]}$ Worryingly, $<50 \%$ of the interns, community-service doctors and medical officers without a Diploma in Anaesthetics (DA), who were surveyed, felt competent performing a GA for CS. During the 2011 - 2013 triennium, ${ }^{[1]} 35 \%$ of the 655686 CSs took place at district hospitals, where there is very little anaesthetic cover by senior staff.

This study shows that there is a large discrepancy in testing levels of block and management of various levels of failure. It further highlights the need for a guideline to be developed for use in SA, where the majority of anaesthetic service providers are relatively junior with limited anaesthetic experience. The failed spinal algorithm should encompass all factors relating to an inadequate regional block and should assist the physician in managing all forms of failure and so reduce maternal and fetal morbidity and mortality.

\section{Conclusion}

There is a need for a standardised assessment of the adequacy of spinal anaesthesia for CS in SA, accompanied by a failed spinal algo- rithm. This may improve obstetric CS outcomes in SA. The recently convened South African Society of Anaesthesiologists Obstetric Anaesthesia Special Interest Society would be the most appropriate body to oversee the creation of an SA guideline.

${ }^{\star}$ Supplemental material. The questionnaire is available from the corresponding author on request.

Acknowledgements. We acknowledge the assistance of Prof. Chris Rout, Dr Eric Hodgson, Prof. Glenda Matthews and Mrs Samantha Jones.

Author contributions. GWJ, RAS and BMB designed the study. GWJ collected the data. GWJ and BMB developed the methodology, performed the analysis and wrote the manuscript.

Funding. Self-funded.

Conflicts of interest. None.

1. Sng BL, Lim Y, Sia AT. An observational prospective cohort study of incidence and characteristics of failed spinal anaesthesia for caesarean section. Int J Obstet Anes 2009;18(3):237-241. https://doi. org/10.1016/.ijoa.2009.01.010

2. Riley E. Regional anesthesia for cesarean section. Tech Region Anesth Pain Manage 2003;7(4):204 212. https://doi.org/10.1016/S1084-208X(03)00038-710.1053/S1084-208X(03)00038-7

3. Kinsella SM. A prospective audit of regional anaesthesia failure in 5080 caesarean sections. Anaesthesia 2008;63(8):822-832. https://doi.org/10.1111/j.1365-2044.2008.05499.x

4. Department of Health. Saving Mothers Report 2008 - 2010. Pretoria: DoH, 2012.

4. Department of Health. Saving Mothers Report 2008 - 2010. Pretoria: DoH, 2012.
5. Eason S, Millar M. City Hospitals Sunderland. Guidelines for inadequate regional anaesthesia 5. Eason S, Millar M. City Hospitals Sunderland. Guidelines for inadequate regional anaesthesia
during LSCS. 2011. httt://www.oaa-anaes.ac.uk/assets/_managed/editor/File/Guidelines/Inadequate $\% 20$ regional\%20block/inadequate_regional_LSCS_Eason_Sunderland.pdf (accessed 23 May 2017).

6. Russell IF. Assessing the block for caesarean section. Int J Obstet Anes 2001;10(2):83-85. https://doi. org $/ 10.1054 /$ ijoa. 2000.0770

7. Russell IF. At caesarean section under regional anaesthesia, it is essential to test sensory block with light touch before allowing surgery to start. Int J Obstet Anes 2006;15(4):294-297. https://doi.org/10.1016/j. ijoa.2006.06.006

8. Fettes PD, Jansson JR, Wildsmith JA. Failed spinal anaesthesia: Mechanisms, management, and prevention. Br J Anaesth 2009;102(6):739-748. https://doi.org/10.1093/bja/aep096

. Royal College of Anaesthetists. Raising the Standard: A Compendium of Audit Recipes. 3rd ed. London: RCoA, 2012 .

10. Farina $Z$, Rout $C$. 'But it's just a spinal': Combating increasing rates of maternal death related to spinal anaesthesia. S Afr Med J 2013;103(2):81-82. https://doi.org/10.7196/samj.6308

11. Gebhardt GS, Fawcus S, Moodley J, Farina Z. Maternal death and caesarean section in South Africa: 1. Gebhardt GS, Fawcus S, Moodley J, Farina Z. Maternal death and caesarean section in South Africa:
Results from the 2011 - 2013 Saving Mothers Report of the National Committee for Confidential Results from the 2011 - 2013 Saving Mothers Report of the National Committee for Confide
Enquiries into Maternal Deaths. S Afr Med J 2015;105(4):287. https://doi.org/10.7196/samj.9351

Accepted 23 February 2017. 